\title{
Per-Norm Based Automotive Headlamp Detection in Hypersepctral Image for Visible Light Communication
}

\author{
Heekang Kim, Sungho Kim* \\ Department of Electronic Engineering, Yeungnam University, Gyungsan-si, Gyeongbuk 712-449, Korea. \\ * Corresponding author. Tel.: +82 53-810-3530; email: sunghokim@ynu.ac.kr \\ Manuscript submitted October 26, 2015; accepted February 10, 2016. \\ doi: 10.17706/ijcee.2016.8.1.12-21
}

\begin{abstract}
This paper introduces a new method which normalizes spectral profile in hyperspectral images. In order to use Visual Light Communication, it should be detected some cars in front of a driver. However, there are some problem in methods using CCD camera for the detection of vehicle. CCD cameras regard the streetlamps as automotive headlamps. In order to solve this problem, hyperspectral camera can be a solution. Because hyperspectral images have hundreds of bands, information of hyperspectral images more than the CCD images. However it is necessary to preprocess hyperspectral image to remove noise and illumination dependency. This paper proposes more effective spectral normalization using Per-Norm. Also it show real experimental results demonstrate the robustness of the proposed method.
\end{abstract}

Key words: Automotive headlamp, hyperspectral image, LED spectral, per-norm, spectral normalization, visible light communication.

\section{Introduction}

Visible Light Communications (VLC) is the name given to an optical wireless communication system by modulating light in the visible spectrum $(400-700 \mathrm{~nm})$, the signal is encoded on top of the illumination light [1]. VLC need to use LED lamps. It can be performed by developing Light Emitting Diode (LED), vehicles are used in an LED headlamp. Especially, the use of white LED. White LED diodes emit in wavelength between $400 \mathrm{~nm}$ and $600 \mathrm{~nm}$ [2]. VLC have some problem. First, LED must be less than or equal to $15 \mathrm{~Hz}$ to satisfy the Nyquist frequency. Therefore this may be slow and humans may be able to recognize such blinking [3]. Also headlamp can communicate incorrectly. Second, VLC just can communicate by using LED lamp. But, there are many others lamp such as Halogen and HID lamp. Therefore initial costs a lot of money to change to LED lamps. Also CCD camera have just three bands. Previous research to get information of vehicle of position was used color segment [4]. It can be regard streetlamps as automotive lamps. Therefore, we research on the other way to get information of vehicle position by spectroscopic using hyperspectral camera. Hyperspectral images are collected at very narrow wavelength intervals [5]. Also hyperspectral image have more information than multispectral image and CCD image. Hyperspectral image combines the features of image and spectroscopy to simultaneously acquire both spatial and spectral data [6]. There are many application from hyperspectral image such as detection damage like spot of fruit in food quality field [7], distinguishing cancer in medical field [8] and target detection in military field [9]. This paper experiment to detect automotive LED headlamp in order to develop active high beam system. There is a big problem, it's that streetlamp also was detected as automotive headlamp. To solve this problem, I proposed normalized 
spectral method which is Percent Normalization (Per-Norm). This paper compares each method (non-normalized, Mean-Norm, Per-Norm) using SAM.

\section{Hyperspectral Image Acquisition System}

The hyperspectral image acquisition system consists of a SPECIM VNIR camera mounted on a rotary tripod. The original image contained a total 1032 bands with a highest spectral resolution, but we used just 258 bands to detect headlamp in order to reduce the number of dimensions. The spectral range was $400-1000 \mathrm{~nm}$. The radiance data was saved in 12-bit binary files. The size of the image $1392 \times 1040$ pixels, but also to reduce data, I just use half data of a total image size like $1053 \times 696$ pixels. Fig. 2 shows the image which composited from RGB bands, the red band is $639 \mathrm{~nm}$, the green band is $549.41 \mathrm{~nm}$ and the blue band is $457.97 \mathrm{~nm}$ in hyperspectral data cube.

Table 1. Specifications of the Hyeprspectral Image Acquisition System

\begin{tabular}{ll}
\hline \hline Item & Specifications \\
\hline Spectral range & $400-100 \mathrm{~nm}(\mathrm{VNIR})$ \\
Spectrograph & ImSpector V10E $30 \mu$ slit, $2.8 \mathrm{~nm}$ spectral resolution \\
Camera & Kappa $1,392 \times 1,040$ pixels, $12 \mathrm{bits}, 11 \mathrm{fps}$, Firewire interface \\
Scanner & Rotational tripod, scan angle : max $160^{\circ}$ \\
\hline \hline
\end{tabular}

Fig. 1 show to install VNIR hyperspectral image acquisition system on the parking lot in Yeungnam University. And distance between camera and automotive headlamp is $400 \mathrm{~m}$.

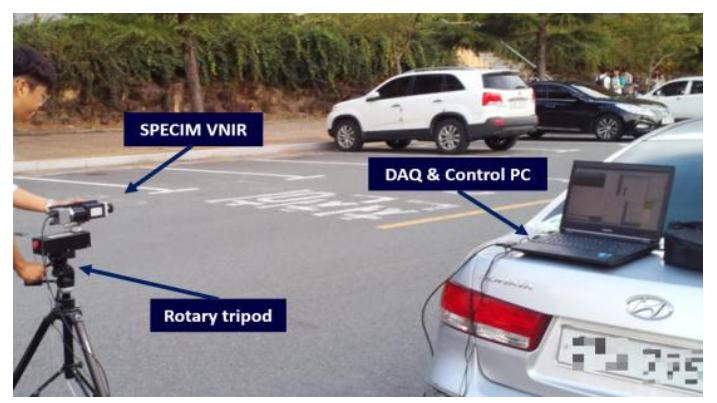

Fig. 1. VNIR hyperspectral image acquisition system.

Headlamp was experimented with Mercedes-Benz's E-class. Headlamps of E-class are made up of LED. Unlike to detect the reflecting light from material, this experiment was conducted to minimize the aperture of hyperspectral camera, since to detect the light from the lamp. Matlab R2015b (The MathWorks Inc., Natick, MA, USA) was used to analyze spectral profile and process headlamp detection.

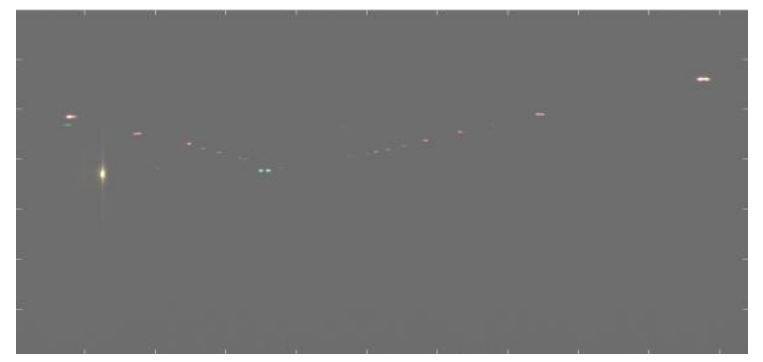

Fig. 2. Image acquired by the hyperspectral camera (night).

Fig. 2 is a composited image from RGB bands in hyperspectral image. Brightness of image was increased to show the detail information in the image. In the small two circles light are headlamps in the $400 \mathrm{~m}$ from hyperspectral camera. 


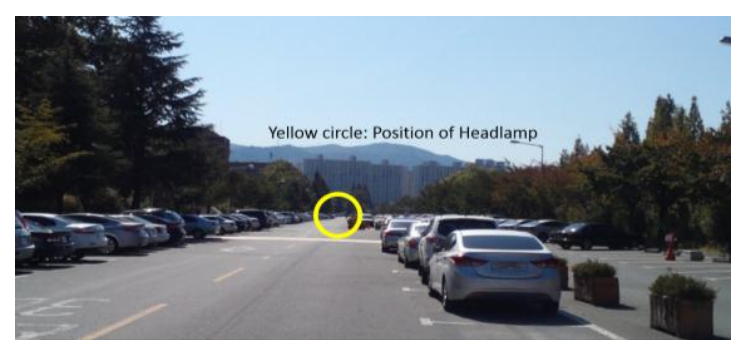

Fig 3. Image acquired by the CCD camera (daytime).

Fig. 3 is a picture taken before sunset on the same place with Fig. 2. There are automotive headlamps in the center of Fig. 3. In addition, there are several clutters such as street lights and parked cars on both sides. We processed cropped images $(186 \times 368)$ from the original images $(1053 \times 696)$ in order to reduce the spatial information.

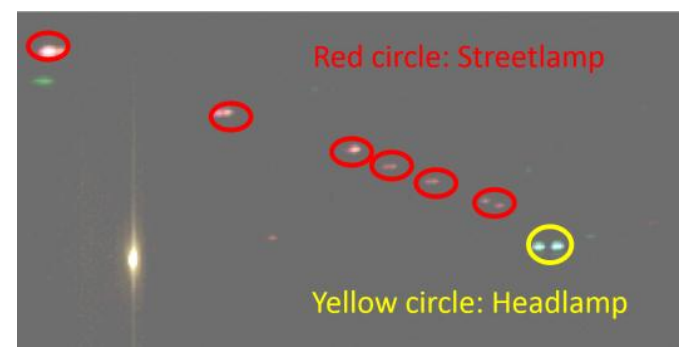

Fig. 4. Headlamp and streetlamp position in cropped image.

Fig. 4 shows a cropped image from the original image. There are six red circle and one yellow circle. The red circles are streetlamp positions and the yellow circle represents the headlamp position. There is a very bright another headlamp which is halogen lamp in the lower left side of Fig. 4. However this light value is meaningless because it is saturated. Therefore, we will detect just the LED headlamp in the yellow circle in Fig. 4. This paper presents how to distinguish other type headlamps.

\section{Propose Per-Norm Based Headlamp Detection}

\subsection{Spectral Profile}

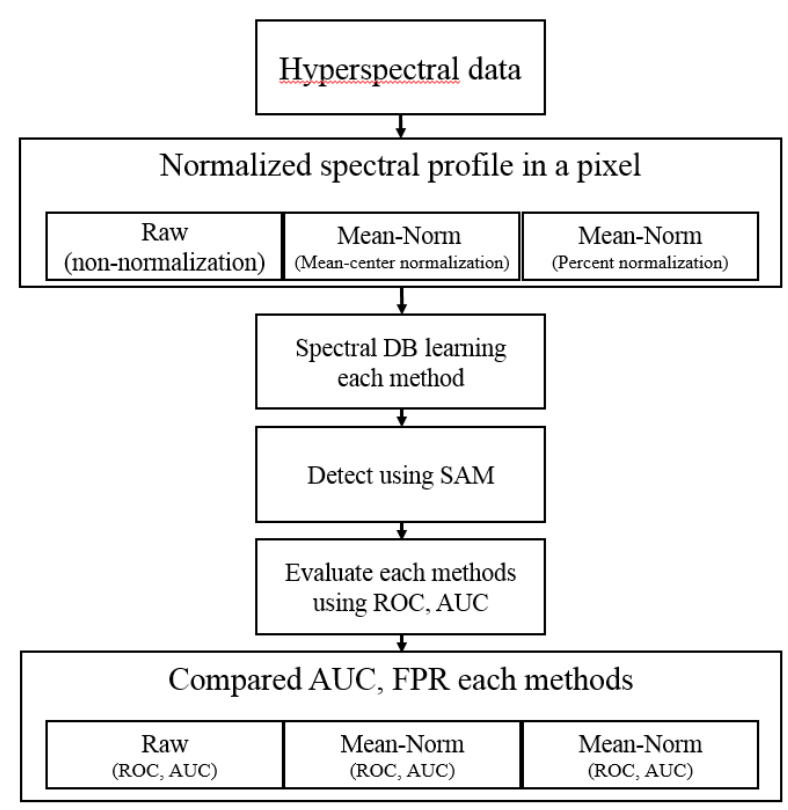

Fig. 5. Block diagram of processing. 
Fig. 5 summarizes the headlamp detection flow including the comparisons between previous methods and the proposed normalization method. Headlamps are detected through the spectral normalization and spectral angle mapper (SAM) detection.

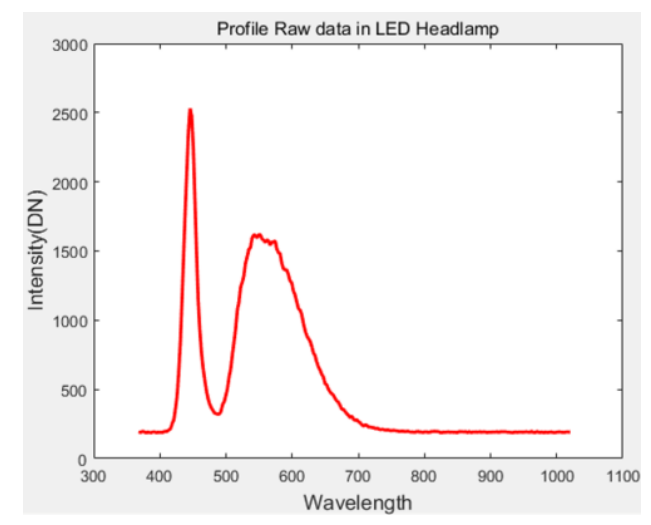

Fig. 6. Spectral profile in headlamp pixel.

Fig. 6 shows the spectral profile of headlamp in the yellow circle of Fig. 4. There is a peak value at $445.71 \mathrm{~nm}$. Because the Light Emitting Diode (LED) do not emit ultraviolet or infrared wavelengths, it is more efficient compared with other light sources such as halogen and HID lamps [10].

Fig. 7 shows the Spectral profile of streetlamp. The streetlamps have different spectral profile information compared with the headlamps.

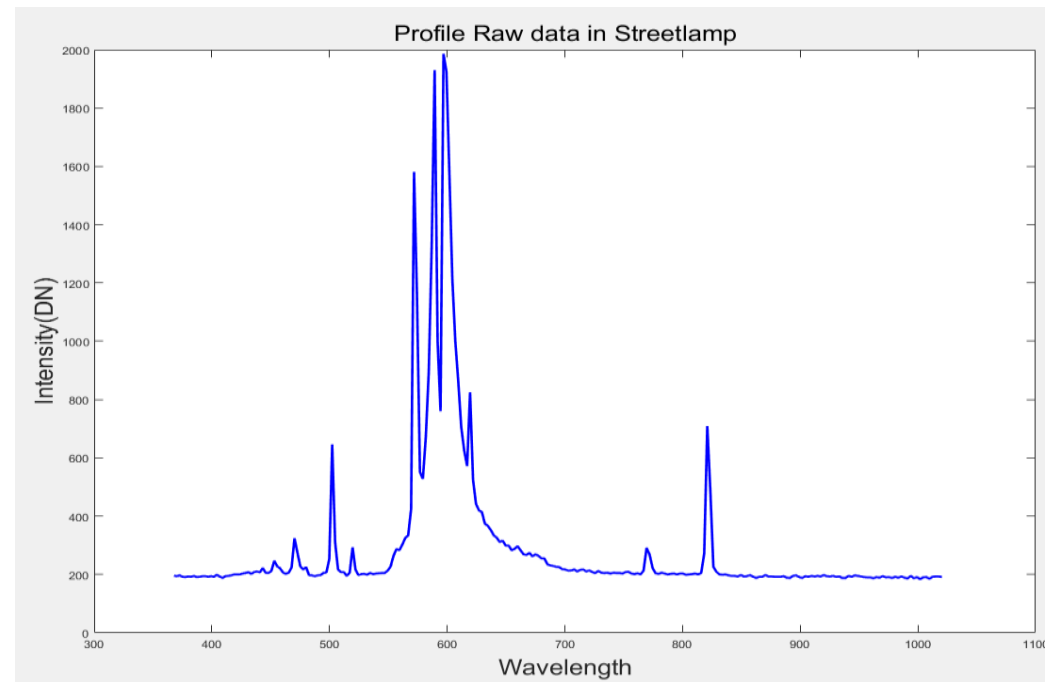

Fig. 7. Spectral profile in streetlamp pixel.

\subsection{Pervious Normalized Spectral Profile Spectral Method}

Normalized technique are used to solve problems generated by different spectral profiles at the same material due to shading and shadow effects [11]. The most common technique is a normalized mean-centered normalization (Mean-Norm) method [12]. It can be expressed as Eq. (1).

$$
I(P, \lambda)=c_{0} \frac{i(P, \lambda)}{\frac{1}{N} \sum_{\lambda=1}^{N} i(P, \lambda)}
$$

where $I(P, \lambda)$ is a normalized spectral profile intensity in $P^{t h}$ pixel at $\lambda^{\text {th }}$ band, $i(P, \lambda)$ is raw spectral 
profile in each wavelength. $N$ is the number of total bands, $c_{0}$ is a scale factor to make a hundred percent [13]. However, it is possible to omit the constant $c_{0}$. In Eq. (1), this intensity value $(I(P, \lambda))$ was divided by mean of Raw spectral profile.

\subsection{Proposed New Normalized Method (Per-Norm)}

Percent Normalization (Per-Norm) is a very effective method in normalizing hyperspectral images. The key idea of Per-Norm is that minimum spectral value in a pixel set to zero and maximum spectral value equals to a hundred. In order to implement the idea, we proposed a new transformation formula. First, remove the minimum value to original (Raw data) value. Second, multiply $c_{0}$ which is scaling to a hundred after removing minimum intensity. It can be expressed as Eq. (2).

$$
\begin{gathered}
I(P, \lambda)=c_{0} \times i(P, \lambda) \\
i^{\prime}(P, \lambda)=i(P, \lambda)-\operatorname{Min}(i(P, \lambda)) \\
c_{0}=\frac{1}{\operatorname{Max}(i(P, \lambda))} \times 100
\end{gathered}
$$

In Eq. (3), (4), minimum intensity of $i(P, \lambda)$ is removed and $c_{0}$ is a constant scaling factor to make a maximum intensity to a hundred.

Let's compare two methods (Mean-Norm, Per-Norm). Fig. 8 shows a new information about advantage of Per-Norm. In case of using Per-Norm, noisy dark current is removed in the raw spectral profile.
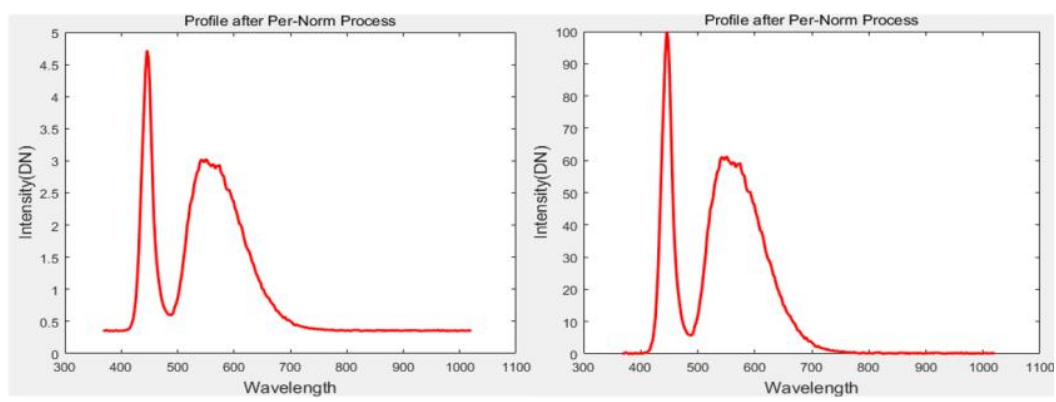

Fig. 8. Compare profile in the two method.

Fig. 9 is a spectral intensity of noise in random pixel. Note that the mean of noise value is around 191 (maximum value is 4095 in Hyperspectral image).

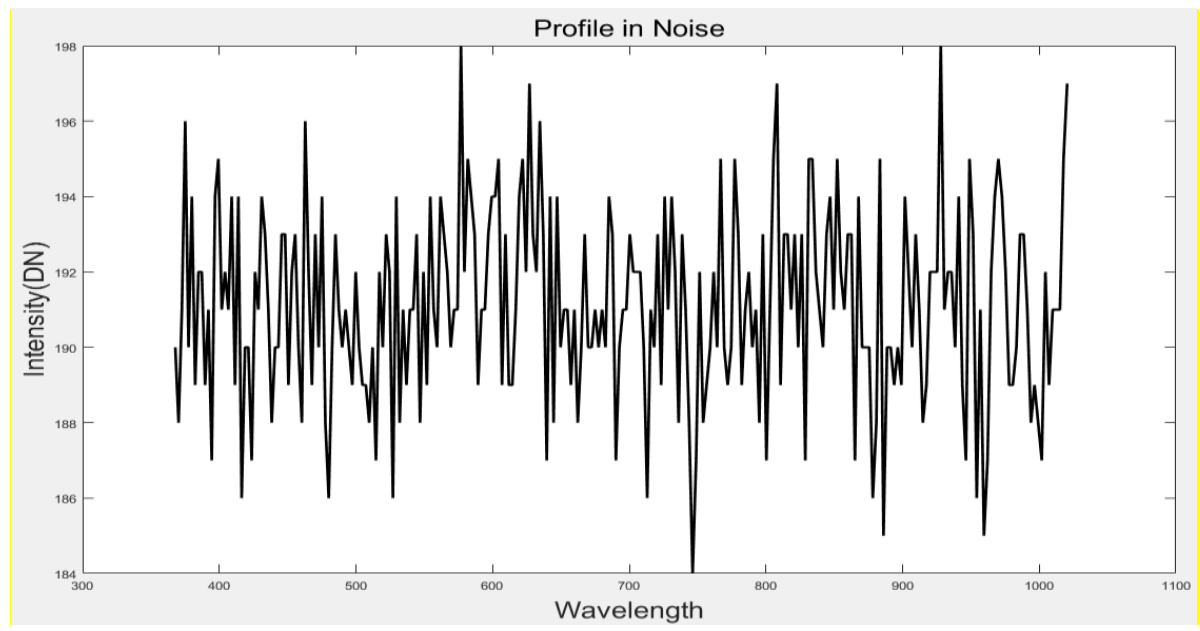

Fig. 9. Spectral intensity in noise pixel. 
The Per-Norm processing can remove the noise intensity. The low intensity corresponding to low information can be removed after removing low intensity value, which can reduce dimensional in hyperspectral image. In addition, targets can be easily detected.

\subsection{Spectral Angle Mapper (SAM)}

The Spectral Angle Mapper (SAM) algorithm is based on the ideal assumption that a single pixel of remotely sensed images represents one certain ground cover material, and can be uniquely assigned to only one ground cover class. The SAM algorithm is just a simple measurement of the spectral similarity between two spectra [14].

In Eq. (5), $\theta(P)$ is spectral angle between a reference spectra and a test spectra of $P^{\text {th }}$ pixel. The reference spectra is a learned profile (LED Headlamp) and the test spectra is a profile of a pixel in the image to check whether it is a headlamp or not.

$$
\theta(P)=\cos ^{-1}\left(\frac{\sum_{n=1}^{N} I_{\text {ref }} \times I_{\text {test }}}{\sqrt{\sum_{n=1}^{N} I_{r e f}^{2}} \times \sqrt{\sum_{n=1}^{N} I_{\text {test }}^{2}}}\right)
$$

Geometrically, the lower the angle (close to 0 degree), each spectra has similar material and the higher the angle (close to 90 degree), the each spectra do not have similar materials as shown in Fig. 10.

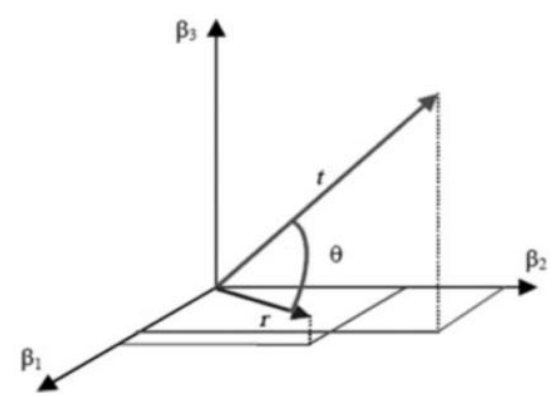

Fig. 10. Describe SAM in three bands Ref. [15].

We use a distance measure $(d)$ based on the angle of SAM as Eq. (6).

$$
d(P)=\frac{\cos \theta(P)}{\max (\cos \theta(\text { Total } P))}
$$

There are three result such as raw data, Mean-Norm and Per-Norm. Each result image uses each reference profile in Fig. 6 and Fig. 8.

\subsection{Distinguish Headlamp and Streetlamp Using SAM}

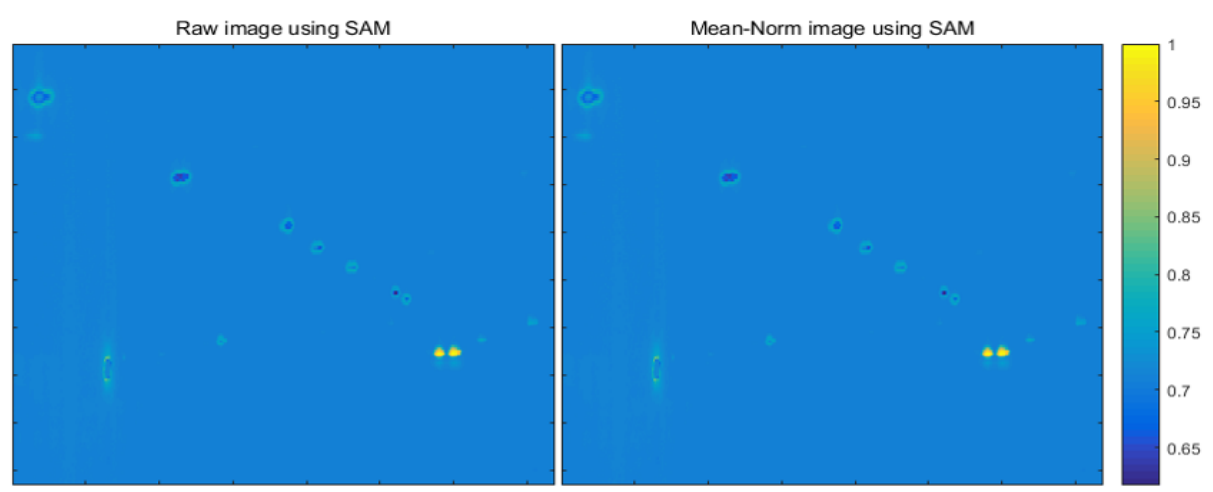

Fig. 11. Compare result raw data and Mean-Norm. 
Fig. 11 shows that the two result images are almost the same. Because Mean-Norm's spectral profile just is obtained by multiplying a constant, mean value of the profile. However, SAM measures angles between a reference profile and a test profile. Therefore, the length of the vector is no meaning, only the angle is significant.

Fig. 12 shows the Per-Norm based SAM results. It can produce better similarity metric than that of raw and Mean-Norm image.

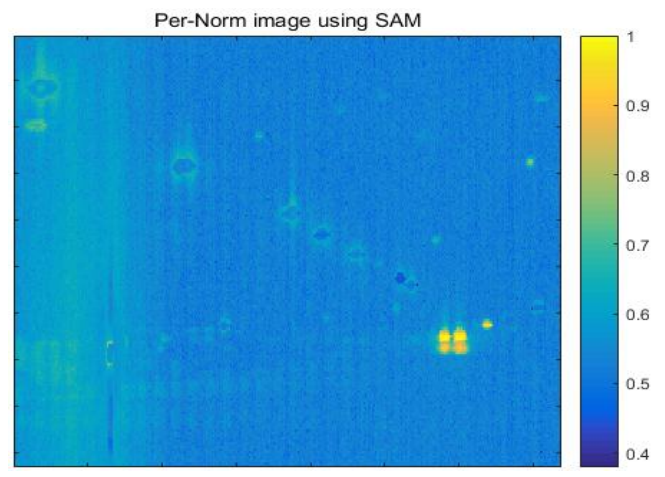

Fig. 12. Result Per-Norm image using SAM.

\section{Experimental Result}

\subsection{Evaluation Performance with Each Method}

In the experiment, detection results can be compared with Area under Curve (AUC) in Receiver Operating Curve (ROC) analysis of detectors. We made a mask image as shown in Fig. 13 in order to compare the detection methods using the ROC and AUC.

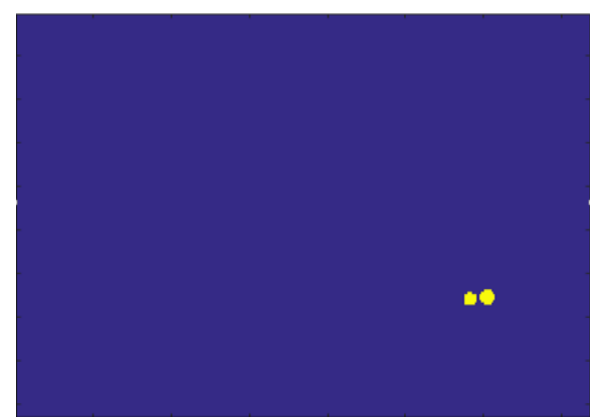

Fig. 13. Given Headlamp position (mask image)

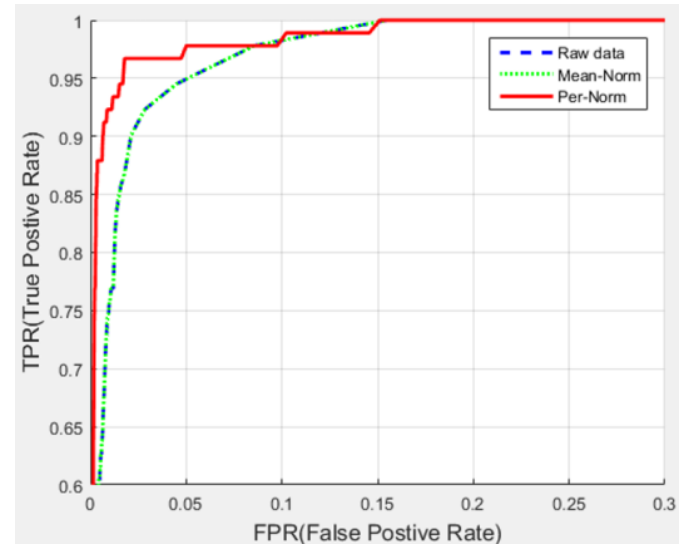

Fig. 14. ROC in each method. 
Fig. 14 is the ROC results of raw data, Mean-Norm and Per-Norm. The Per-Norm proves that it is a better method than the other Mean-Norm in terms of ROC.

Table 2 summarizes three methods in terms of AUC metric. Note that the Per-Norm shows higher AUC value than the Mean-Norm. The AUC of Per-Norm is $0.46 \%$ higher than the Mean-Norm. Other tow methods show high value because the number of target pixel in mask image is very small compared to the total number of pixels. However, Fig. 14 shows the different false positive rate (FPR) value at the same true positive rate (TPR) value in each method.

Table 2. AUC Value in Each Case

\begin{tabular}{lc}
\hline \hline Method & Area Under Curve \\
\hline Raw data & 0.9902 \\
Mean-Norm & 0.9902 \\
Per-Norm & 0.9948 \\
\hline \hline
\end{tabular}

\subsection{Detection Performance in Term of Threshold Sensitivity}

Threshold values changes from 0 to 1 with the interval of 0.001 . When the Detect Rate (DR=TPR) is higher than 0.98 , three result images are compared in terms of the FPR.

Table 3. FPR in Same Threshold (Raw: DR > 0.98)

\begin{tabular}{|c|c|}
\hline Threshold & Raw data (FPR) \\
\hline 0.504 & 0.1549 \\
\hline 0.503 & 0.2644 \\
\hline 0.502 & 0.6141 \\
\hline
\end{tabular}

(a)

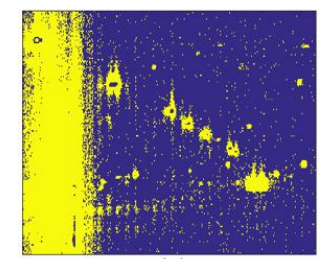

(c)

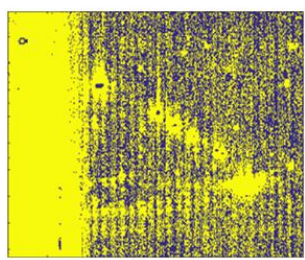

(e)

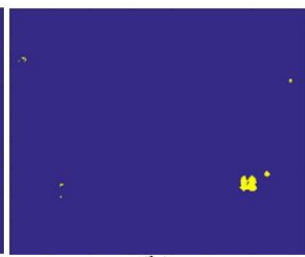

(b)

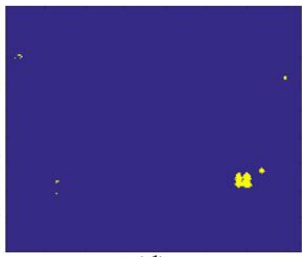

(d)

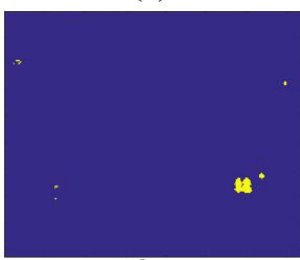

(f)

Fig. 15. Comparison results of Mean-Norm and Per-Norm based detection using SAM distance at threshold $(0.504,0.503,0.502)$.

(a) Mean-Norm at 0.504 threshold; (b) Per-Norm at 0.504 threshold; (thresholds are selected to have the same detection rate); (c) Mean-Norm at 0.503 threshold; (d) Per-Norm at 0.503 threshold; (e) Mean-Norm at 0.502 threshold (f) Per-Norm at 0.502 threshold. 
Fig. 15 shows the compared results of Mean-Norm and Per-Norm when the DR higher than 0.98 with the threshold value of 0.504. In order to compare the difference result between Mean-Norm and Per-Norm precisely, the threshold value is reduced with the interval of 0.001 . As a result, according to Table 3 , the FPR of Per-Norm increase just 0.0001 as the threshold value is changed by 0.001 . However, the FPR of Mean-Norm increase 0.4592 . This result shows that the Per-Norm produces detection results in sensitive to the threshold.

\section{Conclusion}

This paper proposed a Per-Norm based normalized spectral methods in order to detect effective automotive headlamp for visible light communication and active high beam. The proposed Per-Norm has noise removal which helps to detect targets using SAM. The experiments are conducted using SAM distance and evaluated detection performances using ROC and AUC metrics on real headlamp images. According to the results, the prospoed PerNorm method showed better ROC and AUC performance. In addition, the detection performance of the proposed method is less sensitive to threshold values. In the future, we will continue the detection using various headlamps including HID and Halogen. In addition, we will find methods to prevent intensity saturation due to short distance from hyperspectral camera to headlamp.

\section{Acknowledgment}

This research was supported by Basic Science Research Program through the National Research Foundation of Korea (NRF) funded by the Ministry of Science, ICT \& Future Planning (NRF-2014R1A2A2A01002299).

And, this work was supported by Human Resources Program in the Transportation Specialized Lighting Core Technology Development (No. N0001364) granted financial resource from the Ministry of Trade, Industry \& Energy, Republic of Korea.

\section{References}

[1] Arnon, S., (2015). Visible Light Communication. Cambridge University Press.

[2] Ren, S. Y., et al. (2015). Illumination threshold study of retinal security by LED light. Proceedings of 2015 International Conference on Intelligent Systems Research and Mechatronics Engineering.

[3] Yamazato, T., et al. (2014). Image-sensor-based visible light communication for automotive applications. IEEE Communications Magazine, 52(7), 88-97.

[4] Ming, Q., \& Jo, K.-H. (2011). Vehicle detection using tail light segmentation. Proceedings of 2011 6th International Forum on Strategic Technology: Vol. 2.

[5] Konda N., et al. (2015). A prototype on-line AOTF hyperspectral image acquisition system for tenderness assessment of beef carcasses.

[6] Fu, X. P., et al. (2014). Detection of melamine in milk powders based on NIR hyperspectral imaging and spectral similarity analyses. Journal of food Engineering, 124, 97-104.

[7] Rivera, N. V., et al. (2014). Early detection of mechanical damage in mango using NIR hyperspectral images and machine learning. Biosystems Engineering, 122, 91-98.

[8] Du, B., et al. (2012). A spectral dissimilarity constrained nonnegative matrix factorization based cancer screening algorithm from hyperspectral fluorescence images. Proceedings of 2012 International Conference on Computerized Healthcare.

[9] Tiwari, K. C., et al. (2013). Military target detection using spectrally modeled algorithms and independent component analysis. Optical Engineering, 52(2), 026402-026402.

[10] Jung, E. D., \& Lee, Y. L. (2015). Development of a heat dissipating LED headlamp with silicone lens to 
replace halogen bulbs in used cars. Applied Thermal Engineering, 86, 143-150.

[11] Ibrahim, I. (2014). Illumination invariance and shadow compensation on hyperspectral images.

[12] Sun, D.-W. (2010). Hyperspectral Imaging for Food Quality Analysis and Control. Elsevier.

[13] Cheng, X., et al. (2003). NIR/MIR dual-sensor machine vision system for online apple stem-end/calyx recognition. Transactions-American Society of Agricultural Engineers, 46(2), 551-558.

[14] Liu, X. F. (2013). A kernel spectral angle mapper algorithm for remote sensing image classification. Proceedings of 2013 6th International Congress on Image and Signal Processing: Vol. 2.

[15] Ibraheem, I. (2015). Comparative study of maximum likelihood and spectral angle mapper algorithms used for automated detection of melanoma. Skin Research and Technology, 21(1), 84-89.

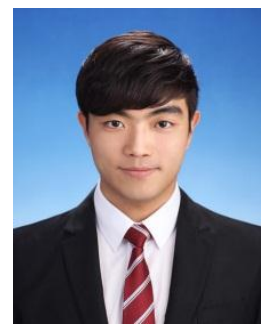

Heekang Kim has received the BC degree in electronic engineering from Yeungnam University. $\mathrm{He}$ is pursuing his $\mathrm{MS}$ degree in electronic engineering from Yeungnam University, Korea. He is working at Advance Visual Intelligence Lab. His research topic are preprocessing to remove noise and normalize spectral, target detection, band selection and object recognition in hyperspectral images.

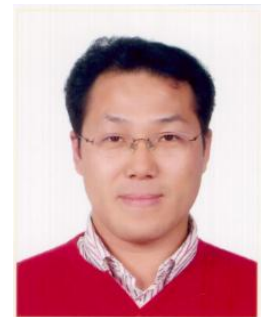

Sungho Kim received the BS degree in electrical engineering from Korea University, Korea in 2000 and the MS, PhD degrees in electrical engineering and computer science from Korea Advanced Institute of Science and Technology, Korea in 2002, 2007, respectively. During 2007 and 2010, he was a senior researcher in the Electro-Optics Laboratory at the Agency for Defense Development (ADD). Since 2010, he has been a professor of electronic engineering at Yeungnam University. His current research interests include hyperspectral image analysis, small infrared target detection, object recognition, human visual perception theory, mobile robot localization, and invariant feature extraction. He is a member of the SPIE, IEEE, the Institute of Electronics Engineering of Korea (IEEK), the Korea Institute of Military Science and Technology (KIMST), Korea Robotics Society (KRS), and ICROS. 